\title{
Association of surfactant protein D with pulmonary metastases from colon cancer
}

\author{
YUKI TAJIMA $^{1}$, MASASHI TSURUTA ${ }^{1}$, HIROTOSHI HASEGAWA ${ }^{1}$, KOJI OKABAYASHI $^{1}$, \\ TAKASHI ISHIDA ${ }^{1}$, MASASHI YAHAGI ${ }^{1}$, AKITSUGU MAKINO ${ }^{1}$, KAORU KOISHIKAWA ${ }^{1}$, \\ SHINGO AKIMOTO ${ }^{1}$, DON D. SIN $^{2}$ and YUKO KITAGAWA ${ }^{1}$ \\ ${ }^{1}$ Department of Surgery, Keio University School of Medicine, Tokyo 160-8582, \\ Japan; ${ }^{2}$ Division of Respiratory Medicine, Department of Medicine, \\ University of British Columbia, Vancouver BC V6Z 1Y6, Canada
}

Received December 23, 2019; Accepted August 4, 2020

DOI: $10.3892 / \mathrm{ol} .2020 .12185$

\begin{abstract}
Surfactant protein D (SP-D) is a member of the collectin family of proteins, which is secreted by airway epithelial cells. SP-D serves an important role in the immune system and in the inflammatory regulation of the lung. SP-D was recently found to suppress lung cancer progression by downregulating epidermal growth factor signaling. However, the relationship between SP-D and pulmonary metastases from colon cancer remains unknown. The present study aimed to determine whether SP-D may suppress the development of the mouse rectal carcinoma cell line, CMT93, in vitro. The present study investigated the effect of SP-D on pulmonary metastases from colon cancer in vivo using SP-D knockout mice. A wound healing assay and cell invasion assay revealed that SP-D suppressed the proliferation, migration and invasion of CMT-93 cells. After injection of CMT-93 cells into the tail vein, SP-D knockout mice were significantly more susceptible to developing pulmonary metastases than C57/BL6 mice (control). Moreover, a novel cell line (CMT-93 pulmonary metastasis; CMT-93 PM) was established from the lesions of pulmonary metastases in C57/BL6 mice following injection of CMT93 into the tail vein. CMT-93 PM exhibited more robust invasion and proliferation compared to CMT93, which was unaffected by exposure to SP-D. A higher incidence of pulmonary metastases was detected following injection of CMT93 PM into the tail vein of C57/BL6 mice compared with CMT-93. Consequently, SP-D may be involved in the pathogenesis of pulmonary metastases from colon cancer.
\end{abstract}

Correspondence to: $\mathrm{Dr}$ Masashi Tsuruta, Department of Surgery, Keio University School of Medicine, 35 Shinanomachi, Shinjyuku-ku, Tokyo 160-8582, Japan

E-mail: masashitsuruta@gmail.com

Key words: surfactant protein D, pulmonary metastasis from colon cancer

\section{Introduction}

Surfactant protein D (SP-D) is a member of the collectin family of proteins secreted by pulmonary epithelial cells. SP-D reduces the surface tension at the alveolar air-liquid interface and prevents the lungs from collapsing at the end of expiration (1). There are four types of surfactant proteins: SP-A, SP-B, SP-C, and SP-D. SP-A and SP-D are hydrophilic proteins that are involved in the immune and inflammatory regulation of the lungs $(2,3)$.

The expression of SP-D is highest in the lungs (4). SP-D is also found in the systemic circulatory system, but its role in this system is unknown (5). Smoking damages the alveolarcapillary barrier, allowing SP-D to leak into the systemic circulatory system. Consequently, SP-D levels decrease in the lung and increase in the serum with smoking (6). A similar phenomenon occurs in other lung inflammatory diseases, such as chronic obstructive pulmonary disease (COPD) (7), idiopathic pulmonary fibrosis (8), and community-acquired pneumonia (9). Lung cancer related to smoking is promoted by pulmonary inflammation (10). This raises the possibility that SP-D may have a regulatory role in the pathogenesis of lung cancer.

Consistent with this notion, SP-D was recently shown to antagonize epidermal growth factor receptor (EGFR) by blocking ligand binding and inhibiting EGFR signaling, which suppressed the proliferation, migration, and invasion of A549 human lung adenocarcinoma cells (11). In vivo lung expression of SP-D is inversely related to the progression of bronchial dysplasia in smokers (12), while a retrospective clinical study revealed that higher serum SP-D levels correlated with better prognosis in selective patients with non-small cell lung cancer (13). Based on these studies, we hypothesized that SP-D could also affect pulmonary metastases from colon cancer.

In this study, we investigated whether SP-D could inhibit the malignant potential of colon cancer cells in vitro and in vivo. We developed an endogenous pulmonary metastasis model of colon cancer in mice and investigated the ability of SP-D to suppress pulmonary metastases from colon cancer in vivo. Furthermore, we established novel cell lines from the 
mouse rectal cancer cell, CMT-93, which are more susceptible to pulmonary metastases, and investigated the impact of SP-D on pulmonary metastases from colon cancer.

\section{Materials and methods}

Cell culture. The mouse CMT-93 rectal carcinoma cell line (CCL-223; ATCC) and the human HCT116 (CCL-247, ATCC) were maintained in Dulbecco's modified Eagle's medium (DMEM; Sigma-Aldrich; Merck KGaA) with $10 \%$ (v/v) fetal bovine serum (FBS) and 1\% penicillin/streptomycin (Thermo Fisher Scientific, Inc.). The cells were cultured at $37^{\circ} \mathrm{C}$ with $5 \% \mathrm{CO}_{2}$.

Wound healing assay. The wound healing assay was conducted using a six-well culture cluster (Corning Incorporated). CMT-93 cells were seeded into the insert in DMEM with $0.1 \%$ (v/v) FCS and grown to confluence (100\%). A wound was introduced into the cells using a $200 \mu \mathrm{l}$ pipette tip and the recombinant Mouse SP-D Protein (R\&D Systems) was added at concentrations of $0,5,10,15$, and $20 \mu \mathrm{g} / \mathrm{ml}$. Twenty-four hours later,cells that had migrated into the scraped areas were counted under a microscope. Five images $(2.8 \times 2.0 \mathrm{~mm})$ per well were captured randomly and the wound areas were calculated using the ImageJ public domain software (14). The same assay using concentrations of 0,5 , and $10 \mu \mathrm{g} / \mathrm{ml}$ of the recombinant Human SP-D Protein (R\&D Systems) was performed using HCT116 cells.

Cell invasion assays. Cell invasion assays were conducted using multiwall 24-well plates (Corning Incorporated) (15). The upper insert was coated with Matrigel $(200 \mu \mathrm{g} / \mathrm{ml}$; cat. no. 354234; Corning Life Sciences) and incubated for $12 \mathrm{~h}$. CMT-93 cells were seeded into the upper insert in DMEM with 10\% (v/v) FBS and 1\% penicillin/streptomycin. In the SP-D group, recombinant SP-D protein was added to the upper insert at a concentration of $0,5,10,15$, and $20 \mu \mathrm{g} / \mathrm{ml}$. The upper insert was placed into the lower outer well in DMEM. The set was incubated at $37^{\circ} \mathrm{C}$ with $5 \% \mathrm{CO}_{2}$ for $36 \mathrm{~h}$. The cells that passed the Matrigel were stained using the diff-quick method and counted with a microscope. The invasion ability was defined as the ratio of the number of the cells that passed through the Matrigel to the number of the cells that did not pass through the Matrigel. The same assay using the concentrations of $0,5,10 \mu \mathrm{g} / \mathrm{ml}$ SP-D was performed using HCT116 cells.

Animals. We used C57BL/6 female mice that were purchased from CLEA Japan. SP-D knockout mice were kindly provided by Dr Don D. Sin in Vancouver, Canada (16). DNA was extracted from tail biopsies of mice using DNeasy blood and tissue kit (Qiagen, Inc.), according to the manufacturer's protocol. GAPDH was identified by PCR using the following primers: Forward, 5'-CGACTTCAACAGCAACTCCCACTC TTCC-3' and reverse, 5'-TGGGTGGTCCAGGGTTTCTTA CTCCTT-3' (Thermo Fisher Scientific, Inc.). SP-D genotypes were identified by multiplex PCR using the following primers: Forward, 5'-TGGTTTCTGAGATGGAGTCGT-3' and reverse, 5'-TGGGGCAGTGGATGGAGTGTGC-3' and 5'-GTGGAT GTGGAATGTGTGCGAG-3' (Thermo Fisher Scientific, Inc.).
PCR was performed with Blend Taq (Toyobo Life Science) under the following conditions: $95^{\circ} \mathrm{C}$ for $15 \mathrm{~min}$, followed by 38 cycles at $94^{\circ} \mathrm{C}$ for $30 \mathrm{sec}, 57^{\circ} \mathrm{C}$ for $30 \mathrm{sec}$ and $72^{\circ} \mathrm{C}$ for $30 \mathrm{sec}$, and a final extension step at $72^{\circ} \mathrm{C}$ for $10 \mathrm{~min}$. The PCR products were separated by $2 \%$ agarose gel electrophoresis and visualized using ethidium bromide. Wild-type (C57BL/6) mice contained $0.4 \mathrm{~kb}$ PCR products but SP-D knockout mice did not (Fig. 1). These animals were acclimatized for at least 7 days before use and were 6 weeks old at the start of the experimental protocol. All animals were housed in a controlled environment at the Keio University School of Medicine under standard temperature and light and dark cycles. All procedures were performed under the approval of the Laboratory Animal Care and Use Committee at the Keio University School of Medicine (approval no. 15006).

Pulmonary metastasis mouse model. The experimental protocol is summarized in Fig. 2. Six-week-old female mice (C57BL/6 mice or SP-D knockout mice) were anesthetized with $2.0 \%$ isoflurane, and $3-5 \times 10^{6}$ cells of CMT-93 in $100 \mu 1$ PBS were injected into the tail vein with a 26 -gauge needle (17). Twenty-five of both the control and SP-D KO mice were used for this experiment. At 8 and 10 weeks following the tail vein injection, the presence of pulmonary metastases was evaluated using an in vivo micro CT (CosmoScan FX, Rigaku) under mild inhalation anesthesia using isoflurane (Fig. 3). Three-dimensional microstructural image data were reconstructed using Tri/3D-BON software (Ratoc System Engineering) (Fig. 3). We compared the number of mice affected by pulmonary metastases, and the number of pulmonary metastases in each mouse. All mice were sacrificed at 12 weeks by exsanguination under systemic anesthesia using $2.0 \%$ isoflurane (Figs. 2 and 3). The lungs were extracted, fixed by injecting methanol $(1 \mathrm{ml})$ through the trachea, and then embedded using paraffin.

Hematoxylin-eosin staining. Lung tissues from the mice were embedded in paraffin and the paraffin blocks were cut into 4- $\mu \mathrm{m}$ thick sections and stained with hematoxylin-eosin. Pathological evaluation for pulmonary metastases was performed using the maximum longitudinal section in the left lobe.

Establishment of the pulmonary-metastasis-prone colon cancer cells. The pulmonary metastasis mouse model was generated as described above. All 15 mice were sacrificed and dissected at 10 weeks. Pulmonary metastases were cut into small pieces on the membrane. Filtered cells from the lung pieces were stirred with DMEM and incubated at $37^{\circ} \mathrm{C}$ with $5 \% \mathrm{CO}_{2}$. Cell culture was performed as described for CMT-93. The cells were grown to confluency and then passaged. After five passages, the cells were diluted to form monoclonal colonies in 96-well culture clusters (Corning Incorporated). Cells were further cultured and passaged more than 10 times. Finally, a novel cell line was established, which we called CMT-93 pulmonary metastasis (CMT-93 PM). The molecular characteristics of CMT-93 PM were assessed by a wound healing assay and invasion assay with or without SP-D. Moreover, CMT-93 PM and CMT-93 were injected into normal mice and the incidence of pulmonary metastases was compared. 


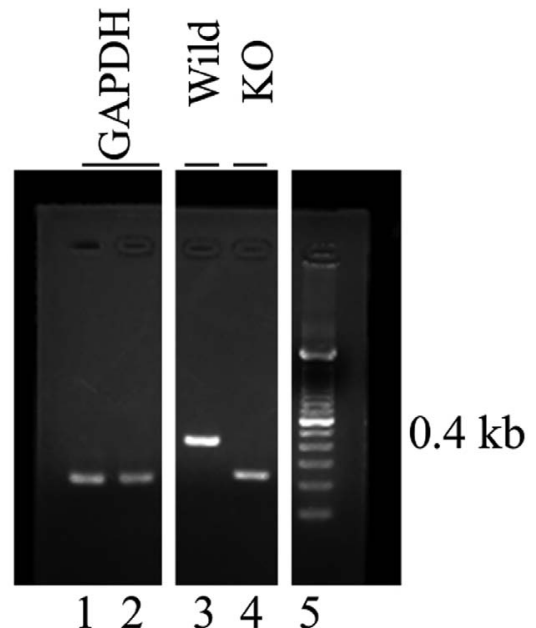

Figure 1. Confirmation of SP-D KO in mice. DNA was extracted from tail biopsies of mice and SP-D genotypes were identified by multiplex PCR Lane 1, GAPDH in wild-type mice; lane 2, GAPDH in SP-D KO mice; lane 3, wild-type mice (0.4 kb PCR product indicated SP-D); lane 4, SP-D KO mice; lane 5, DNA marker. SP-D, surfactant protein D; KO, knockout.

Protocol
6 weeks old mice

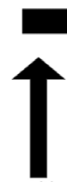

Tail vein

injection
8 weeks 10 weeks 12 weeks

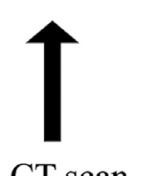

CT scan

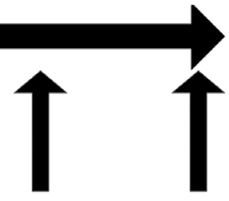

CT scan Sacrifice
Figure 2. Experimental pulmonary metastasis mouse protocol. $3-5 \times 10^{6}$ CMT-93 cells were injected into the tail vein of 6 -week-old female mice (C57BL/6 mice or SP-D knockout mice). At 8 and 10 weeks after the injection, the incidence of pulmonary metastases was evaluated by in vivo micro $\mathrm{CT}$. All mice were killed at 12 weeks immediately after the last CT examination and the lungs were extracted. SP-D, surfactant protein D.

ELISA. ELISA was used to determine the expression of Akt, which is downstream of the EGFR signaling pathway, in CMT-93 and CMT-93 PM. The cells (1×10\%/sample) were serum starved overnight and incubated with SP-D $(10 \mu \mathrm{g} / \mathrm{ml})$ for 2 hours at $37^{\circ} \mathrm{C}$. The cell lysate was prepared and was subjected to ELISA using the Akt (pS473) + total Akt ELISA kits (cat. no. ab126433; Abcam).

Statistical analyses. All results were expressed as the mean value (mean $\pm \mathrm{SE}$ ). All statistical analyses were performed using Stata software (Stata Corp.). $\mathrm{P}<0.05$ was considered to indicate a statistically significant difference. All procedures were performed in triplicate. Differences in the wound healing assay, cell invasion assay and the number of metastases per mouse were statistically analyzed by Mann-Whitney U test or one-way ANOVA. The development of pulmonary metastases in mice was analyzed using $\chi^{2}$ tests.

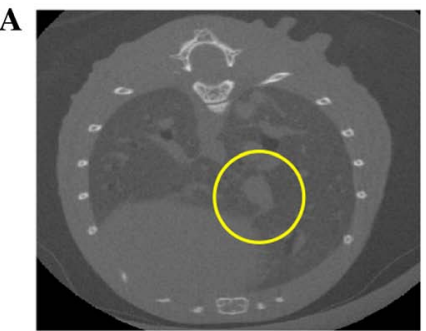

CT scan

C

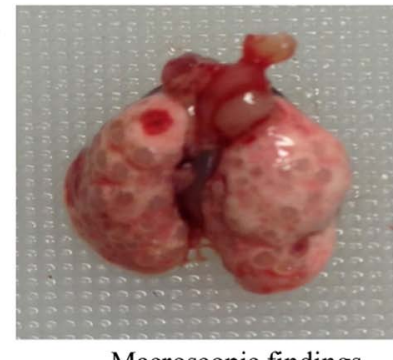

Macroscopic findings

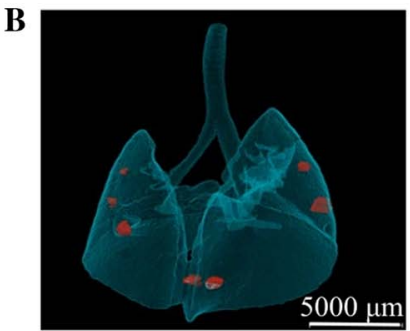

CT reconstruction

D

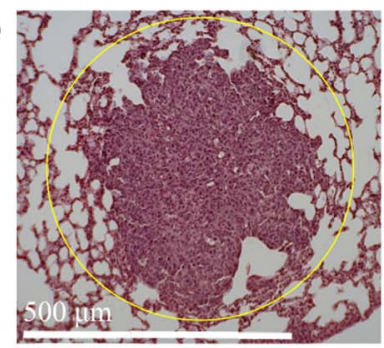

H\&E staining
Figure 3. Pulmonary metastases. (A) A representative two-dimensional image of a CT scan of the pulmonary metastasis mouse model. One nodule was identified in the left lung (circled in yellow). (B) CT reconstruction of the pulmonary metastasis mouse model. Three-dimensional microstructural image data were reconstructed using Tri/3D-BON software. Red colored nodules indicate the pulmonary metastasis. (C) Macroscopic findings of the pulmonary metastasis mouse model. Many nodules were identified in both lungs. (D) H\&E staining of the pulmonary metastasis mouse. $\mathrm{H} \& \mathrm{E}$, hematoxylin-eosin.

\section{Results}

$S P-D$ suppresses the proliferation, migration, and invasion of CMT-93. First, we determined whether the efficacy of SP-D on the malignant potential of CMT-93 is equivalent to primary lung adenocarcinoma cells. SP-D significantly suppressed CMT-93 compared to the control in a wound healing assay, but not in a dose-dependent manner (Fig. 4). In an invasion assay, treatment with SP-D significantly decreased the number of CMT-93 cells that passed through the membrane compared to untreated cells, but not in a dose-dependent manner (Fig. 5). SP-D similarly suppressed migration and invasion ability in human HCT116 cells (Fig. 6).

$S P$-D suppresses pulmonary metastases from colon cancer. We used a pulmonary metastasis mouse model with an SP-D KO to determine if SP-D suppresses pulmonary metastases from colon cancer. The incidence of pulmonary metastases between SP-D KO mice and control C57Bl/6 mice was compared by micro-CT and pathological examination (Fig. 3). Significantly more SP-D KO mice were affected by pulmonary metastases compared to the C57BL/6 mice (Table I) and the average number of metastases per mouse was greater in SP-D KO mice than in C57BL/6 mice in both the CT scans and pathological examinations (Fig. 7).

Establishment of a novel colon cell line, CMT-93 PM. To clarify the mechanism of pulmonary metastasis development and the influence of SP-D, we established a novel colon cancer cell line, CMT-93 PM, which was predisposed to developing 
A

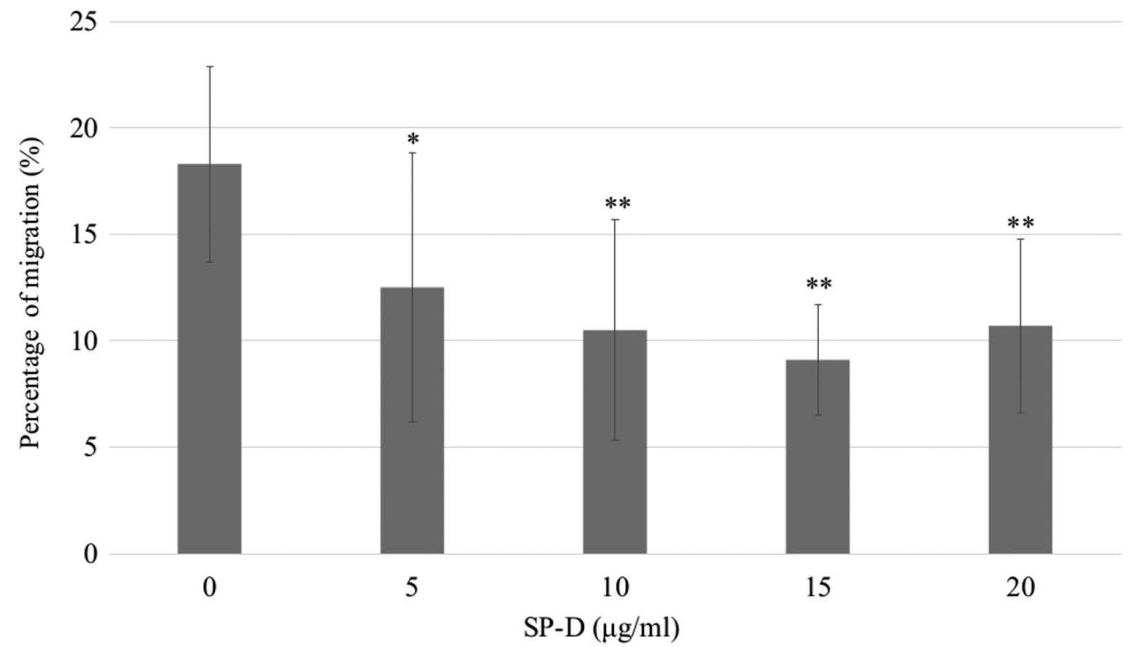

B
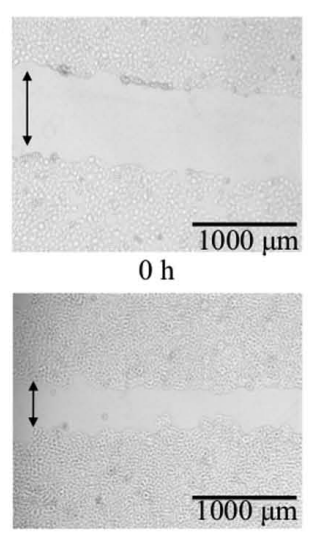

SP-D (-)

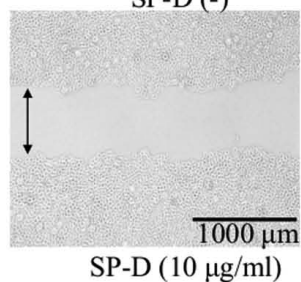

Figure 4. Wound healing assay of CMT-93 cells using SP-D. CMT-93 cells were plated in six-well culture cluster plates. Wounds were introduced using the $200 \mu \mathrm{l}$ pipette tips, and SP-D was added at concentrations of 0,5,10,15 and $20 \mu \mathrm{g} / \mathrm{ml}$. After $24 \mathrm{~h}$, cells that had migrated into the wounded areas were counted under a microscope. Five images $(2.8 \times 2.0 \mathrm{~mm})$ per well were randomly captured, and the wound area was measured using ImageJ. (A) SP-D significantly suppressed the increase in CMT-93 cells compared to the control, but the effect was not dose-dependent. The data shown are mean $\pm \mathrm{SD}$. "P<0.05, ${ }^{* *} \mathrm{P}<0.01$ compared with the control. (B) Representative image of cells at $0 \mathrm{~h}$, the scraped areas without SP-D and the scraped area with $10 \mu \mathrm{g} / \mathrm{ml}$ SP-D. SP-D, surfactant protein D.

A
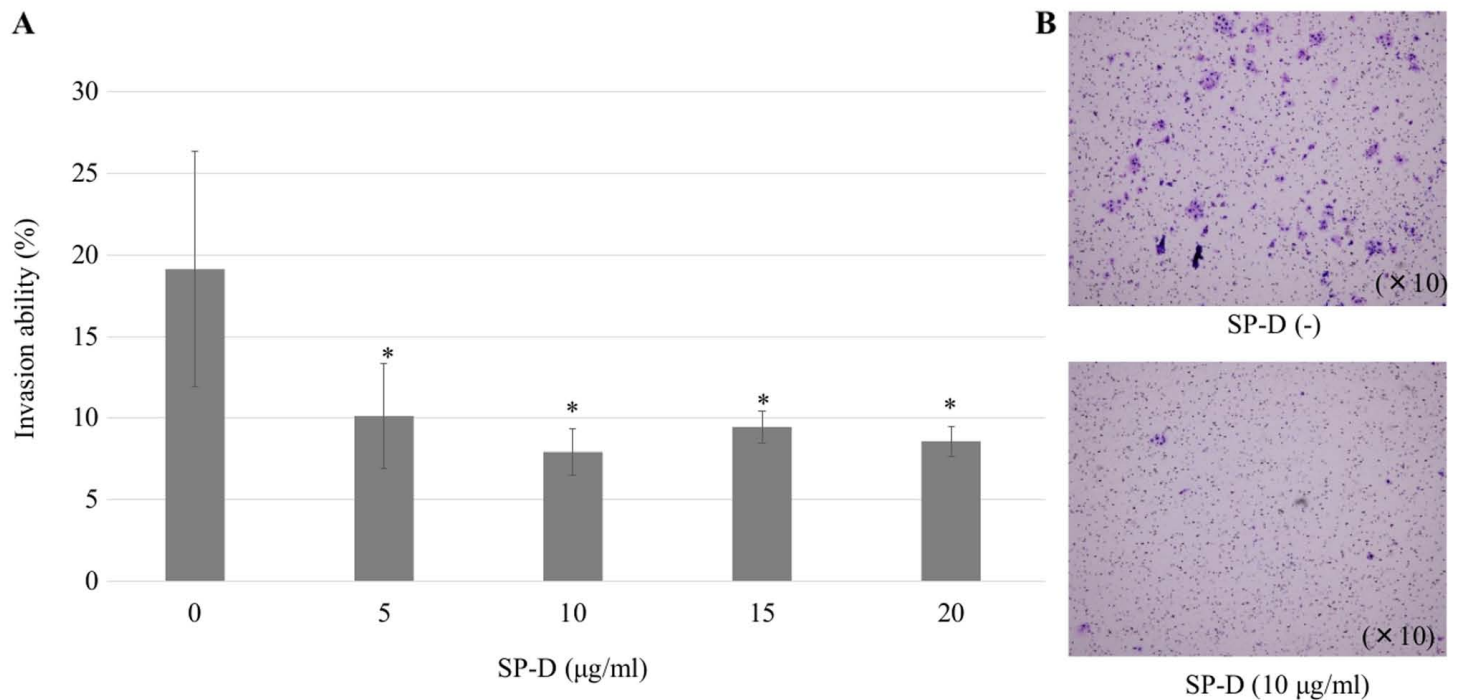

Figure 5. Cell invasion assay of CMT-93 cells using SP-D. CMT-93 cells were plated in 24-well culture cluster plates. The upper insert was coated with Matrigel and incubated for $12 \mathrm{~h}$. CMT-93 cells were seeded in the upper insert. SP-D was also added to the upper insert at a concentration of 0,5, 10, 15 and $20 \mu \mathrm{g} / \mathrm{ml}$. The upper insert was placed in the lower outer well with DMEM. The set was incubated at $37^{\circ} \mathrm{C}$ with $5 \% \mathrm{CO}_{2}$ for $36 \mathrm{~h}$. Cells that passed through the Matrigel were stained using the Diff-Quick method and counted with a microscope. Invasion ability was defined as the ratio of the number of cells that passed through the Matrigel to the number of cells that could not pass through the Matrigel. (A) SP-D significantly decreased the number of CMT-93 cells that passed through the membrane compared to untreated cells (SP-D 5, $10 \mu \mathrm{g} / \mathrm{ml}$ ). The data shown are the mean \pm SD. "P $<0.05$ compared with the control. (B) Representative images of the stained cells (purple) that passed through the membrane without SP-D and images of the stained cells (purple) treated with $10 \mu \mathrm{g} / \mathrm{ml}$ SP-D that passed through the membrane. SP-D, surfactant protein D.

pulmonary metastases. Two clones of CMT-93 PM, called CMT-93 PM (1) and CMT-93 PM (2), were identified. A wound healing assay showed that the proliferative capacity of CMT-93 PM was higher than CMT-93 (Fig. 8). An invasion assay showed that the SP-D-induced suppression of invasion ability was weaker in CMT-93 PM (1) than that of CMT-93PM. Moreover, SP-D reversely enhanced the invasion ability of CMT-93 PM (2), while it was lower than that of CMT-93 without SP-D (Fig. 9). In vivo, we confirmed that injection of CMT-93 PM (2) cells into the tail vein resulted 
Table I. Number of mice with pulmonary metastasis, evaluated using CT scan and pathological examination.

\begin{tabular}{lccc}
\hline Method & SP-D KO mice, $\mathrm{n}=24(\%)$ & C57BL/6 mice, $\mathrm{n}=23(\%)$ & P-value \\
\hline CT scan & $11(45.8)$ & $4(17.4)$ & 0.037 \\
Pathological examination & $15(62.5)$ & $5(21.7)$ & 0.005 \\
\hline
\end{tabular}
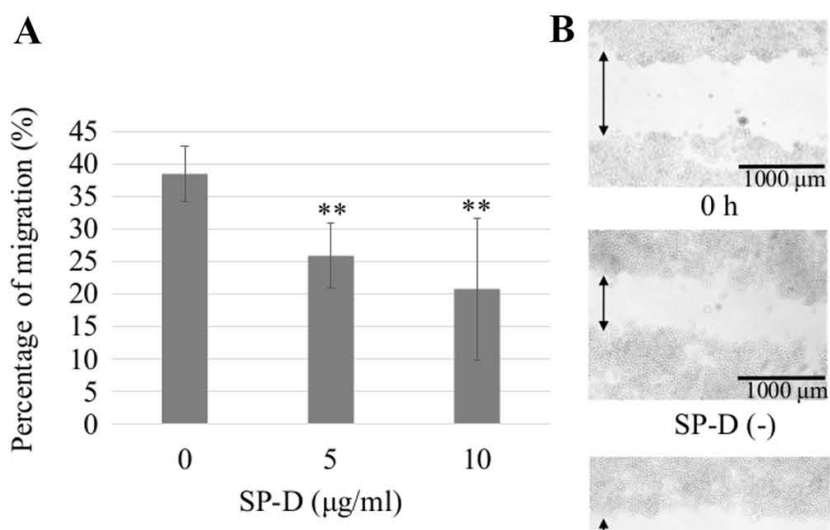

$\mathbf{C}$

D
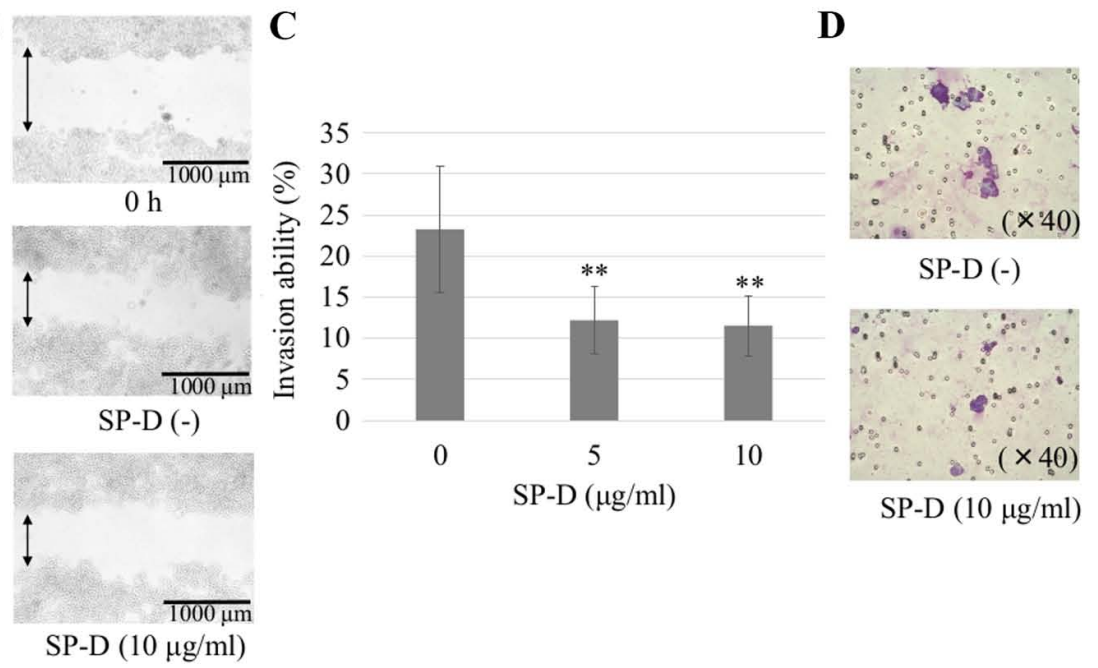

Figure 6. Wound healing assay and cell invasion assay of HCT-116 cells using SP-D. (A) Wound healing assay of HCT-116 cells. SP-D significantly suppressed the increase in HCT-116 cells. The data shown are mean \pm SD. ${ }^{* *} \mathrm{P}<0.01$ compared with the control. (B) Representative images of cells at $0 \mathrm{~h}$, of the scraped areas without SP-D and of the scraped areas with SP-D $(10 \mu \mathrm{g} / \mathrm{ml})$. The line shows the length of the wound. (C) Cell invasion assay of HCT-116 cells. SP-D significantly suppressed the increase in HCT-116 cells. The data shown are mean \pm SD. ${ }^{* *} \mathrm{P}<0.01$ compared with the control. (D) Representative image of the untreated stained cells (purple) that passed through the membrane without SP-D and of the stained cells (purple) that passed through the membrane treated with SP-D $(10 \mu \mathrm{g} / \mathrm{ml})$. SP-D, surfactant protein D.
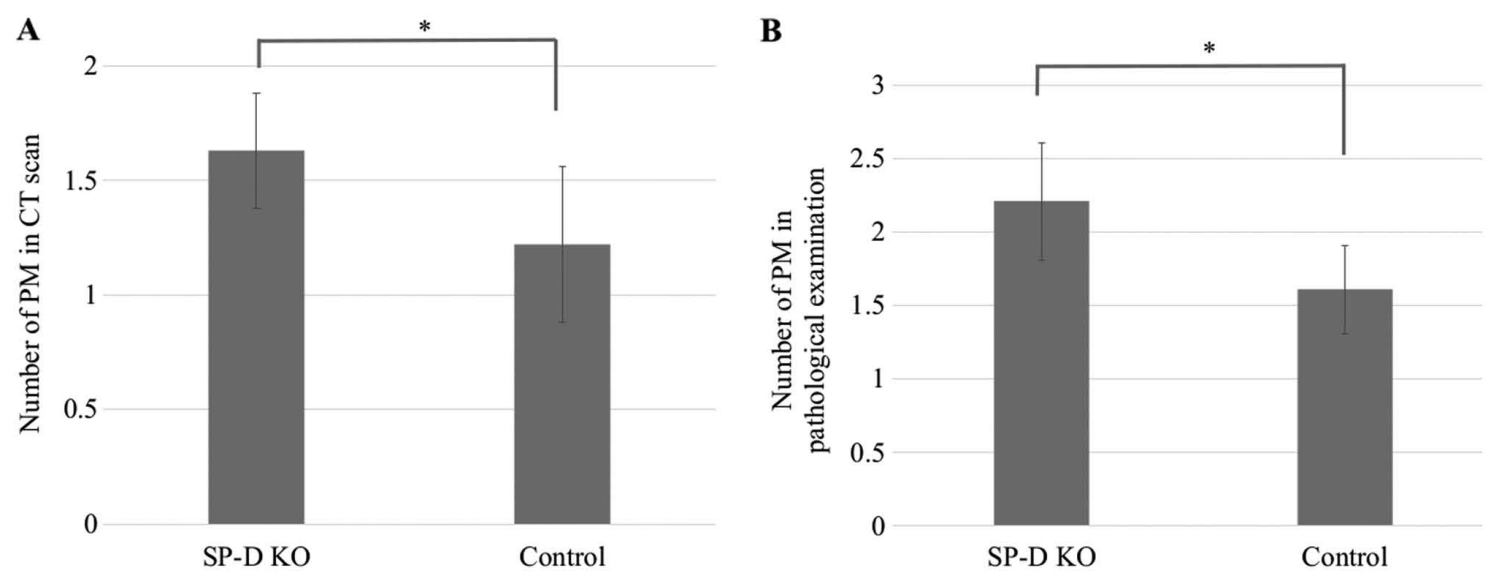

Figure 7. Comparison of PM between SP-D KO and control mice. (A) CT scan analysis shows that a significantly greater number of pulmonary metastases were present in SP-D KO mice than C57BL/6 mice (1.63 vs. 1.22; $\mathrm{P}=0.045)$. The data shown are mean \pm SD. ${ }^{*} \mathrm{P}<0.05$ compared with the control. (B) Pathological examination showed that a significantly greater number of pulmonary metastases were present in SP-D KO mice than in C57BL/6 mice (2.21 vs. 1.61; $\mathrm{P}=0.019)$. The data shown are mean $\pm \mathrm{SD}$. ${ }^{*} \mathrm{P}<0.05$ compared with the control. SP-D, surfactant protein D; PM, pulmonary metastases; KO, knockout.

in the development of significantly more pulmonary metastases compared to CMT-93 (80.0 vs. $20.0 \%$; $\mathrm{P}=0.025$ ).

Change of Akt due to SP-D. SP-D suppressed the malignant potential through the EGF-EGFR signaling pathway, in which SP-D played antagonist to the EGFR and downregulated its signal according to a previous study. Thus, we evaluated the change in Akt level by SP-D in CMT-93 and CMT-93PM (2), which were the most resistant cells against SP-D. Both cell types demonstrated a decrease in Akt level due to SP-D (Fig. 10). CMT-93PM showed a relatively small change in Akt level due to the SP-D treatment compared to CMT-93 (0.20 vs. $0.11 ; \mathrm{P}=0.10)$ (0.24 vs. 0.18 ; $\mathrm{P}=0.88)$. 
$\mathbf{A}$

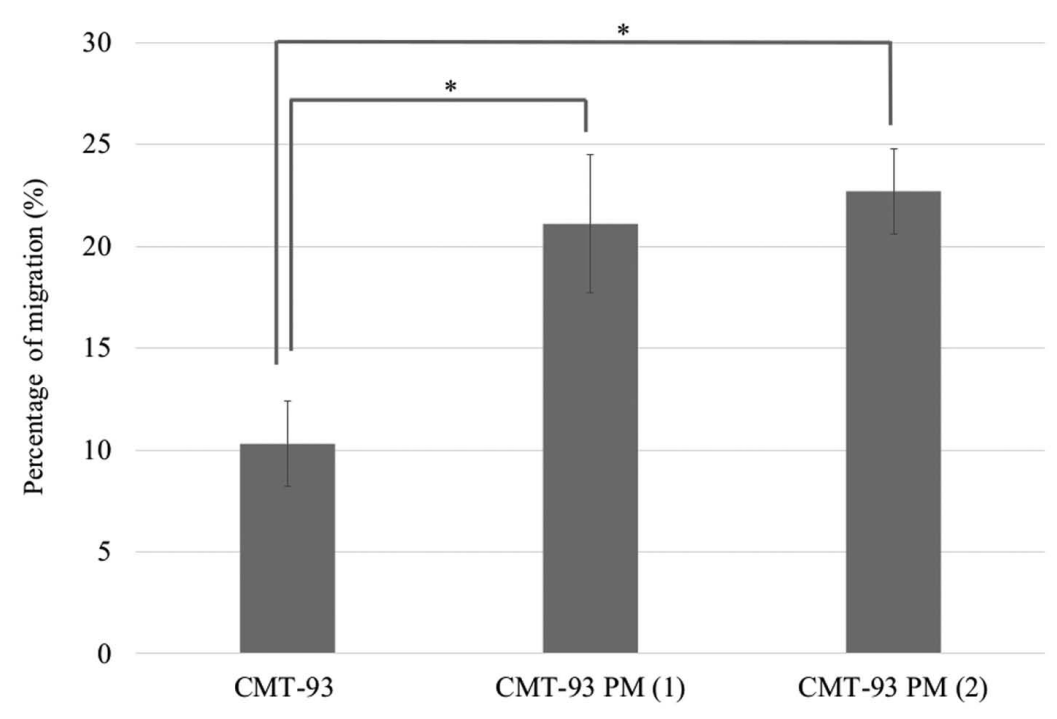

B
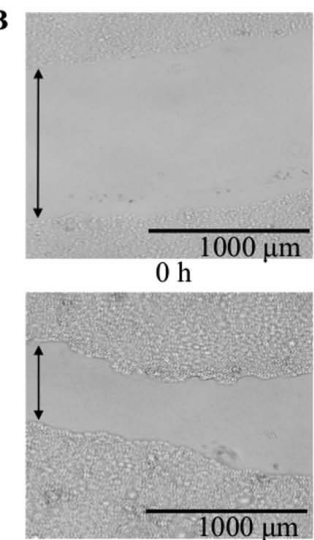

CMT-93

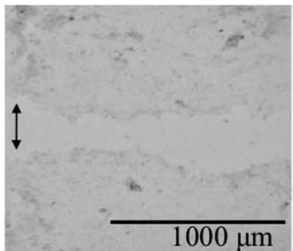

CMT-93 PM (2)

Figure 8. Wound healing assay of CMT-93 cells and CMT-93 PM with SP-D. (A) The proliferative capacities of CMT-93 PM (1) and (2) were higher than those of CMT-93 cells following treatment with SP-D $(10 \mu \mathrm{g} / \mathrm{ml})$. The data shown are mean \pm SD. ${ }^{*} \mathrm{P}<0.05$. (B) Representative image of cells at $0 \mathrm{~h}$, and of the scraped areas of CMT-93 and CMT-93 PM (2). The line shows the length of the wound. SP-D, surfactant protein D; CMT-93 PM, CMT-93 pulmonary metastases.

A

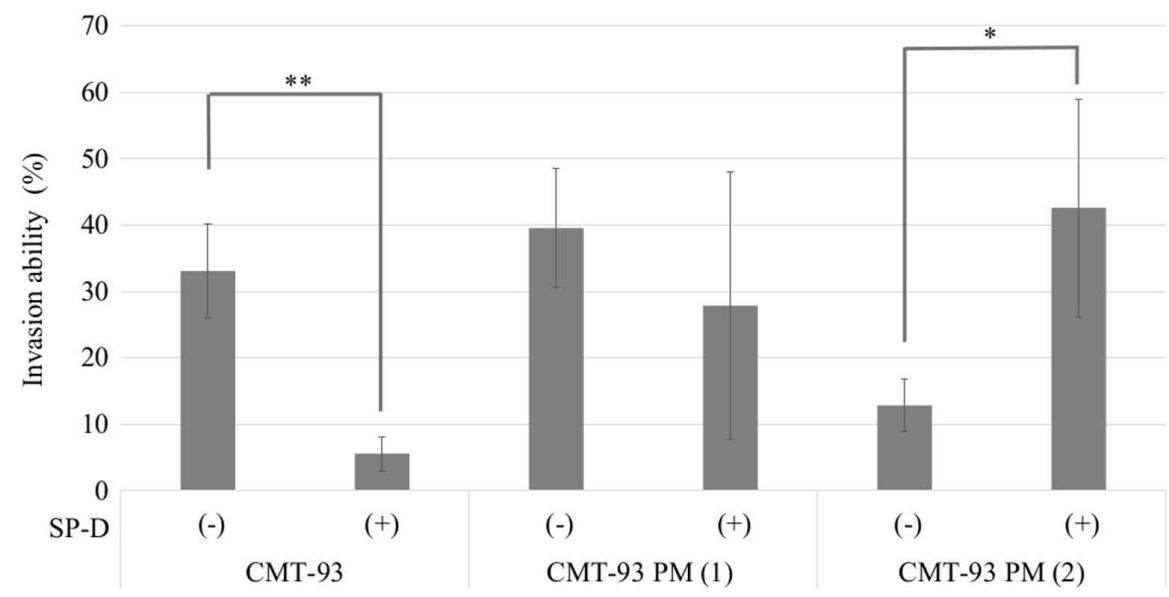

B

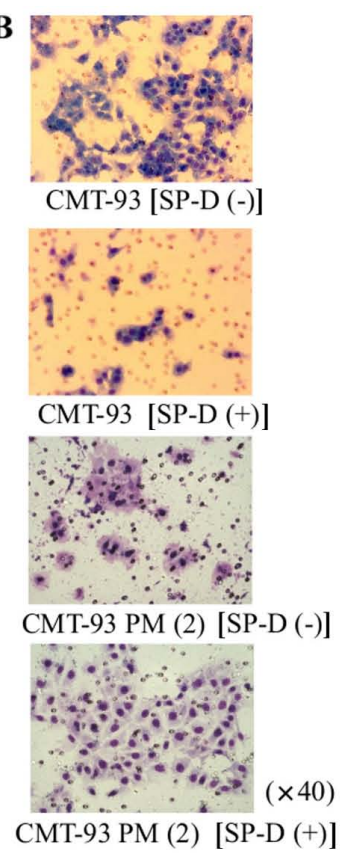

Figure 9. Cell invasion assay of CMT-93 cells and CMT-93 PM using SP-D. (A) Both CMT-93 PM cell lines showed resistance to treatment with $10 \mu \mathrm{g} / \mathrm{ml}$ of the SP-D protein, whereas SP-D suppressed the invasion ability of CMT-93 cells. CMT-93 PM (2) enhanced the invasion capacity due to the SP-D treatment $(\mathrm{P}=0.016)$. The data shown are mean $\pm \mathrm{SD} .{ }^{*} \mathrm{P}<0.05,{ }^{* *} \mathrm{P}<0.01$ compared with the control. (B) Representative images of the stained CMT-93 cells (purple) that passed through the membrane treated without SP-D and with SP-D, and images of the stained CMT-93 PM (2) cells (purple) that passed through the membrane treated without SP-D and with SP-D. SP-D, surfactant protein D; CMT-93 PM, CMT-93 pulmonary metastases.

\section{Discussion}

SP-D is an important protein for the maintenance of the alveolar structure and management of the immune system in the lung (1-3). In addition, leakage of SP-D from the lung into the systemic circulatory system causes increased levels of serum SP-D. Therefore,
SP-D is a surrogate marker of COPD (7). Moreover, SP-D is an antagonist of EGFR and suppresses the signals downstream of EGFR, which inhibits the progression of primary lung adenocarcinoma cells (11). We hypothesized that SP-D suppresses pulmonary metastases from colon cancer in the same manner. In this study, we found that SP-D impacts pulmonary metastases from colon cancer. 


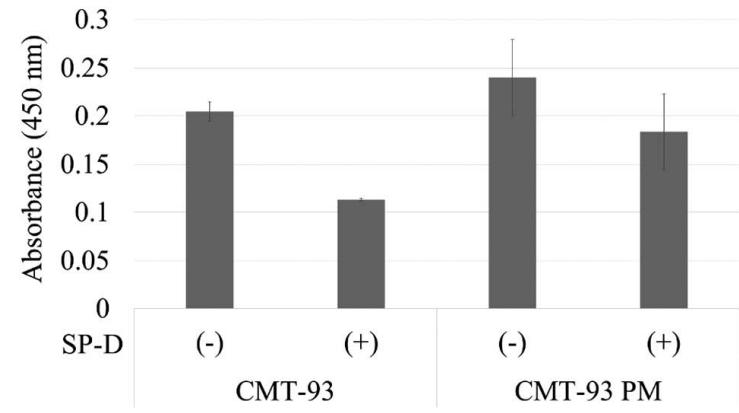

Figure 10. The expression of Akt of CMT-93 cells and CMT-93 PM by ELISA. CMT-93 and CMT-93 PM (1x10\%/sample) were serum starved overnight and incubated with SP-D $(10 \mu \mathrm{g} / \mathrm{ml})$ for 2 hours at $37^{\circ} \mathrm{C}$. The cell lysate was prepared and the expression of Akt was calculated by ELISA using the Akt (pS473) + total Akt ELISA kits. CMT-93PM showed a relatively small change in Akt level due to the SP-D treatment compared to CMT-93 ( 0.20 vs. $0.11 ; \mathrm{P}=0.10)$ ( 0.24 vs. $0.18, \mathrm{P}=0.88)$. $\mathrm{SP}-\mathrm{D}$, surfactant protein $\mathrm{D}$; CMT-93 PM, CMT-93 pulmonary metastases.

We found that SP-D suppressed the progression of CMT-93 colon cancer cells, which was comparable to a previous study of primary lung adenocarcinoma cells (11). Therefore, we hypothesized that SP-D would also play a role in the pathogenesis and development of pulmonary metastases from colon cancer. After the injection of CMT-93 into the tail vein of mice, significantly larger and more numerous pulmonary metastases were found in SP-D KO mice compared to the control. Consequently, loss of SP-D in the lung enhanced and exacerbated the pulmonary metastases from colon cancer through increased cell proliferation.

As the influence of SP-D in tissues other than the lung was removed, we determined that the serum SP-D protein level was $6.89 \pm 1.4 \mathrm{ng} / \mathrm{ml}$. Our result was almost the same as one previously reported $(18,19)$. Thus, the influence of SP-D in serum could be negligible during the development of pulmonary metastasis in our model.

Smoking causes inflammation in the lung, which damages the membrane between the alveolar and vasculature and allows SP-D to leak into the systemic circulatory system. Consequently, the SP-D levels decrease in the lung and increase in the circulatory system. Our previous study demonstrated that smoking is a risk factor for pulmonary metastasis from colon cancer (20) and SP-D is a potential player. The reduction of SP-D in the lung caused by smoking may also be a risk factor for pulmonary metastasis from colon cancer. The results of this study support our hypothesis that SP-D in the lung suppresses the development and progression of pulmonary metastasis from colon cancer by blocking EGFR signals (11).

In addition, we established a novel pulmonary metastasis prone cell line, CMT-93 PM, to increase the impact of SP-D on pulmonary metastasis. Interestingly, CMT-93 PM was more resistant to SP-D than CMT-93. Therefore, resistance to SP-D is required for development of pulmonary metastases. CMT-93 PM increased the incidence of pulmonary metastases following the injection of the cells into the tail vein. Similarly, more pulmonary metastases were found in SP-D KO mice than $\mathrm{C} 57 \mathrm{Bl} / 6$ mice.

We used a micro-CT scan and 3D rearrangement to evaluate the pulmonary metastasis. In addition, we performed two examinations within 2 weeks, which allowed for more accurate identification of pulmonary metastases from vasculature or minor bronchus. We also performed a pathological evaluation, which confirmed the radiological results.

Since SP-D inhibits EGFR signaling in A549 human lung adenocarcinoma cells, the same pathway is likely to be involved in the growth of pulmonary metastases from colon cancer. In this study, we did not directly check SP-D binding with the EGFR, but we initially confirmed expression of EGFR in both CMT-93 and CMT-93PM. We also evaluated the change in the Akt level to determine whether the EGFR signaling pathway was involved in the different behavior of SP-D between CMT93 and CMT-93PM. As a result, both cells demonstrated a decrease in Akt level due to SP-D, but the change in CMT-93PM was smaller. Therefore, downregulation of the EGF-EGFR signaling pathway might contribute to the resistance to SP-D. SP-D is a collectin protein that binds cytokine ligands. Further experiments including identification of its ligands would contribute to our results.

Although further experiments are needed, our results are promising for managing pulmonary metastasis from colorectal cancer. In the future, SP-D might be a feasible biomarker for monitoring, and restitution of SP-D into the lung could be a novel strategy for preventing or treating pulmonary metastases, though the effect of restoration of SP-D on this mouse model needs to be evaluated.

In conclusion, we demonstrated that SP-D suppressed the growth of pulmonary metastases from colon cancer in vivo and in vitro using a pulmonary metastasis mouse model.

\section{Acknowledgements}

Not applicable.

\section{Funding}

No funding was received.

\section{Availability of data and materials}

The datasets used and/or analyzed during the current study are available from the corresponding author on reasonable request.

\section{Authors' contributions}

YT and MT participated in the study design and coordination, and drafting of the manuscript. MY, AM, KK and SA performed the experiments and participated in the acquisition of data. KO and TI participated in the design of the study and performed the statistical analysis. HH, DDS and YK conceived the study, participated in its design and coordination, and helped to draft the manuscript. All authors read and approved the final manuscript and agree to be accountable for all aspects of the research.

\section{Ethics approval and consent to participate}

All the procedures were performed under the approval of the Laboratory Animal Care and Use Committee at Keio University School of Medicine (approval no. 15006). 


\section{Patient consent for publication}

Not applicable.

\section{Competing interests}

The authors declare that they have no competing interests.

\section{References}

1. Johansson $\mathrm{J}$ and Curstedt T: Molecular structures and interactions of pulmonary surfactant components. Eur J Biochem 244: 675-693, 1997.

2. Crouch E and Wright JR: Surfactant proteins A and D and pulmonary host defense. Ann Rev Physiol 63: 521-524, 2001

3. Kuroki Y, Takahashi M and Nishitani C: Pulmonary collectins in innate immunity of the lung. Cell Microbiol 9: 1871-1879, 2007.

4. Mori K, Kurihara N, Hayashida S, Tanaka M and Ikeda K: The intrauterine expression of surfactant protein $\mathrm{D}$ in the terminal airways of human fetuses compared with surfactant protein A Eur J Pediatr 161: 431-434, 2002.

5. Sørensen GL, Hjelmborg Jv, Kyvik KO, Fenger M, Høj A, Bendixen C, Sørensen TI and Holmskov U: Genetic and environmental influences of surfactant protein $\mathrm{D}$ serum levels. Am J Physiol Lung Cell Mol Physiol 290: L1010-L1017, 2006.

6. Sin DD, Pahlavan PS and Man SF: Surfactant protein D: A lung specific biomarker in COPD? Ther Adva Respir Dis 2: 65-74, 2008

7. Ozyurek BA, Ulasli SS, Bozbas SS, Bayraktar N and Akcay S: Value of serum and induced sputum surfactant protein-D in chronic obstructive pulmonary disease. Multidiscip Respir Med 8: 36, 2013

8. Greene KE, King TE Jr, Kuroki Y, Bucher-Bartelson B, Hunninghake GW, Newman LS, Nagae H and Mason RJ: Serum surfactant proteins-A and -D as biomarkers in idiopathic pulmonary fibrosis. Eur Respir J 19: 439-446, 2002.

9. Leth-Larsen R, Nordenbaek C, Tornoe I, Moeller V, Schlosser A, Koch C, Teisner B, Junker P and Holmskov U: Surfactant protein D (SP-D) serum levels in patients with community-acquired pneumonia. Clin Immunol 108: 29-37, 2003.

10. Smith CJ, Perfetti TA and King JA: Perspectives on pulmonary inflammation and lung cancer risk in cigarette smokers. Inhal Toxicol 18: 667-77, 2006.

11. Hasegawa Y, Takahashi M, Ariki S, Asakawa D, Tajiri M, Wada Y, Yamaguchi Y, Nishitani C, Takamiya R, Saito A, et al: Surfactant protein D suppresses lung cancer progression by downregulation of epidermal growth factor signaling. Oncogene 6: 828-845, 2014.
12. Sin DD, Man SF, McWilliams A and Lam S: Surfactant protein D and bronchial dysplasia in smokers at high risk of lung cancer. Chest 134: 582-588, 2008.

13. Umeda Y, Hasegawa Y, Otsuka M, Ariki S, Takamiya R, Saito A, Uehara Y, Saijo H, Kuronuma K, Chiba H, et al: Surfactant protein $\mathrm{D}$ inhibits activation of non-small cell lung cancer-associated mutant EGFR and affects clinical outcomes of patients. Oncogene 36: 6432-6445, 2017

14. Grada A, Otero-Vinas M, Prieto-Castrillo F, Obagi Z and Falanga V: Research techniques made simple: Analysis of collective cell migration using the wound healing assay. J Invest Dermatol 137: e11-e16, 2017.

15. Zhao X, Yang W, Pei F, Ma W and Wang Y: Downregulation of matrix metalloproteinases contributes to the inhibition of cell migration and invasion in HepG2 cells by sodium valproate. Oncol Lett 10: 531-535, 2015.

16. Hirano Y, Choi A, Tsuruta M, Jaw JE, Oh Y, Ngan D, Moritani K, Chen YR, Tam S, Li Y, et al: Surfactant protein-D deficiency suppresses systemic inflammation and reduces atherosclerosis in ApoE knockout mice. Cardiovasc Res 113: 1208-1218, 2017.

17. Eberting CL, Shrayer DP, Butmarc J and Falanga V: Histologic progression of B16 F10 metastatic melanoma in C57BL/6 mice over a six week time period: Distant metastases before local growth. J Dermatol 31: 299-304, 2004.

18. Gaunsbaek MQ, Rasmussen KJ, Beers MF, AtochinaVasserman EN and Hansen S: Lung surfactant protein D (SP-D) response and regulation during acute and chronic lung injury. Lung 191: 295-303, 2013.

19. Hansen S, Schmidt V, Steffensen MA, Jensen PH, Gjerstorff M, Thiel S and Holmskov U: An enzyme-linked immunosorbent assay (ELISA) for quantification of mouse surfactant protein D (SP-D). J Immunol Methods 31: 75-85, 2008.

20. Yahagi M, Tsuruta M, Hasegawa H, Okabayashi K, Toyoda N, Iwama N, Morita S and Kitagawa Y: Smoking is a risk factor for pulmonary metastasis in colorectal cancer. Colorectal Dis 19: O322-O328, 2017.

This work is licensed under a Creative Commons Attribution-NonCommercial-NoDerivatives 4.0 International (CC BY-NC-ND 4.0) License. 